\title{
Compound Control Strategy for MDF Continuous Hot Pressing Electrohydraulic Servo System with Uncertainties and Input Saturation
}

\author{
Zhu Liang-kuan, ${ }^{1}$ Wang Zi-bo, ${ }^{1}$ and Liu Ya-qiu ${ }^{2}$ \\ ${ }^{1}$ School of Electro-Mechanical Engineering, Northeast Forestry University, Harbin 150040, China \\ ${ }^{2}$ School of Information and Computer Engineering, Northeast Forestry University, Harbin 150036, China \\ Correspondence should be addressed to Zhu Liang-kuan; zhulk@126.com
}

Received 27 June 2016; Revised 17 October 2016; Accepted 10 November 2016

Academic Editor: Francisco Gordillo

Copyright (C) 2016 Zhu Liang-kuan et al. This is an open access article distributed under the Creative Commons Attribution License, which permits unrestricted use, distribution, and reproduction in any medium, provided the original work is properly cited.

A compound control strategy is investigated for Medium Density Fiberboard (MDF) continuous hot pressing electrohydraulic servo system (EHSS) with uncertainties and input saturation. Firstly, a hyperbolic tangent function is applied to approximate saturation nonlinearity in the system. And thus the mathematical model is continuous and differentiable. Subsequently, the slab thickness tracking controller is constructed by using a dynamic surface control (DSC) method, which introduces first-order low-pass filters to calculate derivatives of virtual control input in each step. Compared with the conventional backstepping controller, complexity of the design procedure is alleviated obviously. Moreover, a composite disturbance of uncertainties and input saturation is estimated by a nonlinear disturbance observer for compensation of the control law. Finally, an appropriate Lyapunov function is chosen to prove that all signals of the closed-loop system are semiglobally uniformly ultimately bounded and the tracking error converges to zero asymptotically. Numerical simulation results are also exhibited to authenticate and validate the benefits of the proposed control scheme.

\section{Introduction}

With the increasing contradiction between supply and demand of timber resource, Medium Density Fiberboard (MDF) plays a significant role in the wood based panel market, owing to its favorable physical characteristics and excellent mechanical properties [1]. At present, continuous hot pressing electrohydraulic servo system (EHSS) is widely used to produce MDF. Hot pressing, as a core process of producing MDF, determines its final density, thickness, shape, and so on [2]. Particularly, gauge thickness is the key to obtain the highquality MDF. Therefore, precise thickness control should be investigated for the MDF continuous hot pressing EHSS.

Unfortunately, both uncertainties and input saturation, which are inherent in the MDF continuous hot pressing EHSS, have a great influence on the control performance, degrading the precision of slab thickness. On one hand, uncertainties lie in system parameter perturbations and external disturbances. Due to the existence of temperature and pressure, some system parameters such as oil bulk modulus $\beta_{e}$ and oil liquid density $\rho$ may vary violently as the time goes by [3]. Simultaneously, external disturbances, consisting of steam pressure in the slab and environmental factors, have an undesirable impact on the EHSS. On the other hand, input saturation is one of inevitable nonlinearities from the actuator limitation [4]. Once the control input exceeds the upper voltage, the actuator action is invariable. Then, the electrohydraulic servo valve fails to supply the sufficient oil to the hydraulic cylinder. It will deteriorate the transient performance and accuracy of the EHSS severely. What is worse, it probably leads to instability. Accordingly, actuator saturation is not only a practical phenomenon, but also a theoretical problem when a precise control law is designed.

For the uncertainties in the EHSS, sliding mode control (SMC) attracted considerable attention [5-8], owing to its insensitiveness to the parameter perturbations and external disturbances during the sliding motion. However, chattering 
problem emerged in the system when the discontinuous sliding mode is applied, resulting in the extra power consumption and unmodeled high-frequency dynamics. Reference [5] introduced a varying boundary layer into the SMC to suppress the chattering. Combining adaptive law with SMC, [6] removed the assumption that bounds of uncertainties should be known and chattering phenomenon. By virtue of the universal approximation, fuzzy self-tuning mechanism [7] and adaptive Radial Basis Function (RBF) neural network [8] were brought into the SMC. Moreover, since the EHSS is always treated as a three-order or high integral cascade system, backstepping technique [9-11], known as a systematic and recursive design methodology with the flexibility, was widely utilized.

Nevertheless, the aforementioned literatures ignored the effect of input saturation in the EHSS. To handle the saturation constraints, a number of approaches have been presented and employed into different fields so far. In [12], an antiwindup method was proposed for a class of linear systems subject to actuator saturation. Reference [13] developed an adaptive model predictive controller (MPC) for nonlinear system in presence of saturation. By means of command filters, a constrained backstepping strategy is applied for flight control [14]. Although the abovementioned researches coped with the saturation problem in various systems, rigorous proof and general design procedures could not be guaranteed. Recently, Wen et al. introduced a smooth function to approximate the input saturation and an auxiliary system with the Nussbaum function to compensate for the effect arising from saturation nonlinearity in [15]. It argued that the transient performance depended on some certain design parameters in an explicit way. Thereafter, [16] constructed an adaptive neural network (ANN) controller by Gaussian error function to represent the input saturation. It ensure that the tracking error could converge to a small neighborhood around an origin.

It is supposed to point out that tackling both uncertainties and saturation for the EHSS is more challenge than taking one single problem into consideration with some specified control approach. There are still some common problems as follows: (1) When the SMC or backstepping method is used to design the controller, they need to calculate the high-order derivatives of reference signals and virtual control in each subsystems which may aggravate the calculation burden. It is unconducive to implement in practice. (2) As mentioned above, some researchers introduced an auxiliary system [15] and adaptive neural network [16] to eliminate the saturation effects, causing complexity of the designed controller.

Note that the main task is simplifying the design procedure from two aspects. One is alleviating the calculation burden. In particular, the existing backstepping technique in the EHSS gives rise to "explosion of differential terms" obviously, due to its repeated derivatives of virtual control inputs and signal references. The complexity grows drastically as the order increases. Therefore, a dynamic surface control (DSC) strategy was presented by Swaroop et al. in [17], applying several first-order low-pass filters to calculate the derivatives of virtual control inputs. Then, the burdensome calculation is avoided effectively.
The other is reducing the complexity of controller as possible. Although control algorithm with adaptive law, artificialintelligence based method, and auxiliary systems might get perfect robustness and antisaturation properties, they also introduced quantities of extra parameters, such as adaptive law parameters and NN weights [18]. Such parameters will further aggravate the complexity of the controller, especially in presence of uncertainties and input saturation. Hence, nonlinear disturbance observer- (NDO-) based control strategy is popular because of its simple formation and less design parameters [19]. Some scholars view the model uncertainties, external disturbances, and saturation nonlinearities as a composite disturbance term and adopt a NDO to estimate and compensate it appropriately. References [2024] suggested that NDO-based controllers have advantages on the robustness and compensation of saturation without many design parameters.

Except for the problem of uncertainties and input saturation, there are also some other factors which will have an impact on the MDF slab thickness, like unreliable communication links [25], input delay [26], sensor fault, and limited communication capacity [27].

Inspired by the work mentioned above, a compound control strategy is investigated for the MDF continuous hot pressing electrohydraulic servo system (EHSS) with uncertainties and input saturation. The controller chooses the DSC approach to realize the tracking control and the NDO to compensate the composite disturbance term, consisting of uncertainties and saturation nonlinearities. The control motivation is used to cancel the influence which aroused the composite disturbance and guarantees a precise thickness control performance with lower calculation burden.

The contribution can be summarized as follows:

(1) Due to the existence of input saturation in the system, the MDF slab thickness tracking control performance is impacted. A hyperbolic tangent function is introduced into the MDF continuous hot pressing EHSS system mathematic model to approximate the saturation nonlinearities. And thus a continuous differentiable model is obtained to ensure that the DSC approach can be applied.

(2) With the help of two first-order low-pass filters, the derivatives of virtual control inputs are obtained. Compared with conventional backstepping controller, the complexity of design in the DSC method is alleviated. Meanwhile, the burdensome calculation is avoided which is conducive to the engineering implement.

(3) We develop a NDO to estimate the composite disturbance term of uncertainties and saturation nonlinearity. It effectively compensates for the designed control law and guarantees the MDF slab thickness precision.

The organization of the rest paper is as follows. The description of hot pressing process and the EHSS model is given in Section 2. In Section 3, a compound controller is established with the DSC technique and the NDO. The stability analysis is presented in Section 4. Simulation results 


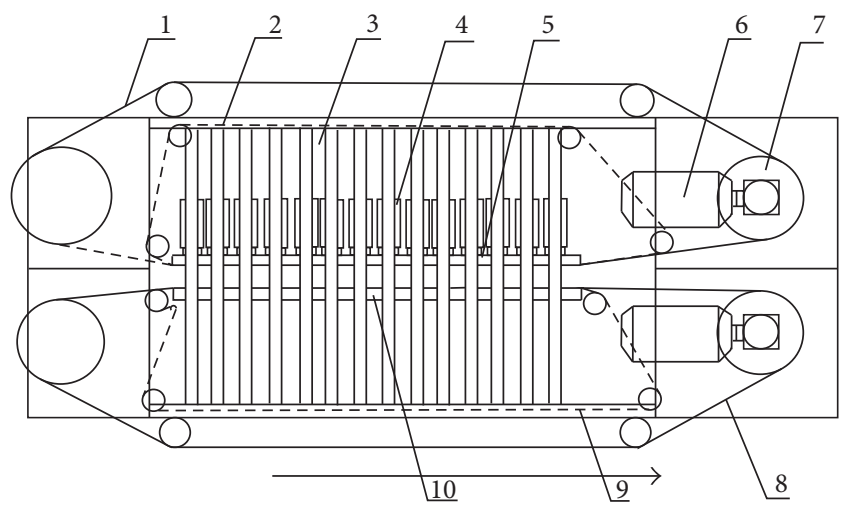

Figure 1: Configuration of continuous hot pressing machine. 1: upper steel conveyor, 2: upper chain blanket, 3: rank, 4: hydraulic cylinder, 5: upper platen, 6: electric motor, 7: driven rolling wheel, 8: lower steel conveyor, 9: lower chain blanket, and 10: lower platen.

are performed to demonstrate the effectiveness of the proposed strategy in Section 5. Finally, the paper is concluded in Section 6.

\section{Process and System Description}

2.1. Hot Pressing Process. Hot pressing is an essential process of the flat pressing way to produce MDF. Namely, high temperature and high pressure are supplied to MDF slab after preloading process. The chemical components of fiber are degraded in the presence of high temperature. Then, the fiber activity is excited. At the same time, bonding force is formed among the fiber. The aim of high pressure is to suppress the rebounded force inside and discharge the steam. Hence, the fiber can interweave tightly [28]. In addition, the MDF slab is pressed to the specified thickness under the pressure.

Particularly, gauge thickness is the key to obtain the high-quality MDF. Slab thickness is determined in a fixingthickness phase, which is mainly about the final MDF figuration. With temperature and pressure in a reasonable range, discharging the left steam in the slab is the main task to avoid the defects such as surface bubbling and lamination. Nevertheless, due to the existence of steam pressure, thickness deviation is prone to appear. In the thicker area, steam cannot be discharged under the same pressure. Therefore, precise gauge control of MDF slab thickness is particularly significant at the fixing-thickness stage to cancel the deviation immediately.

2.2. The Hot Pressing EHSS System. Configuration of the continuous hot pressing machine is depicted in Figure 1. A MDF slab is carried into an inlet by steel conveyor belts whose driving force is supplied from a couple of driven rolling wheels. Then, it reaches on a pair of steel platens, accomplishing the hot pressing process. The pressure is provided by hundreds of hydraulic cylinders and the temperature is transferred by the heated oil in the cylinder [28].

At present, MDF hot pressing control system mainly adopts electrohydraulic servo system (EHSS), shown in
Figure 2. MDF continuous hot pressing has two control types, which are the pressure control and the position control. In this paper, the position control is researched. The objective of controller is tracking the desired position reference signal to realize a precise gauge control of MDF slab thickness.

In practice, as the current limitation of servo valve, the control input is constrained. In the other words, the servo valve is on longer work than usual, when the control input increases, so called input saturation. Owing to the restricted control input, the desired position reference signal will not be tracked accurately, decreasing the precision of the MDF slab thickness.

2.3. Problem Statement and Mathematic Model. Three basic equations of a four-way valve controlled hydraulic cylinder power mechanism are given as follows, where the detailed deduction refers to [29].

$$
\begin{aligned}
Q_{L} & =c_{d} \omega x_{v} \sqrt{\frac{1}{\rho}\left(p_{s}-p_{L} \operatorname{sgn}\left(x_{v}\right)\right)} \\
Q_{L} & =A \frac{d y}{d t}+C_{\mathrm{tc}} p_{L}+\frac{V_{t}}{4 \beta_{e}} \frac{d p_{L}}{d t} \\
A p_{L} & =m \frac{d^{2} y}{d t^{2}}+B_{c} \frac{d y}{d t}+K y+F,
\end{aligned}
$$

where $Q_{L}$ is load flow, $c_{d}$ is sliding valve flow coefficient, $\omega$ is sliding valve area gradient, $\rho$ is liquid density, $p_{s}$ is oil supply pressure, $p_{L}$ is load pressure, $x_{v}$ represents the displacement of valve spool, $A$ is effective area of piston, $y$ represents piston displacement, $C_{\mathrm{tc}}$ is the total leakage coefficient, $V_{t}$ is oil cylinder volume, $\beta_{e}$ is elastic modulus of oil, $m$ is load gross mass, $B_{c}$ is viscous damping coefficient, $K$ is load spring stiffness, and $F$ represents the external load force.

The servo amplifier is equivalent to the proportion, and the link between input current of servo valve and displacement of valve spool is also proportional; hence they can be described as follows:

$$
\begin{aligned}
K_{\mathrm{sv}} & =\frac{x_{v}}{i} \\
K_{a} & =\frac{i}{u},
\end{aligned}
$$

where $K_{\mathrm{sv}}$ is servo valve gain, $K_{a}$ is servo amplifier gain, $i$ is input current of servo valve, and $u$ is output of the controller.

State equation of EHSS is yielded from (1) and (2). States $x_{1}=y, x_{2}=\dot{y}$, and $x_{3}=\ddot{y}$ represent displacement, speed, and acceleration of the hydraulic cylinder piston motion, respectively. As a result, the mathematic model is described as

$$
\begin{aligned}
\dot{x}_{1} & =x_{2} \\
\dot{x}_{2} & =x_{3} \\
\dot{x}_{3} & =f(x)+g\left(x_{v}\right) u+d \\
y & =x_{1},
\end{aligned}
$$




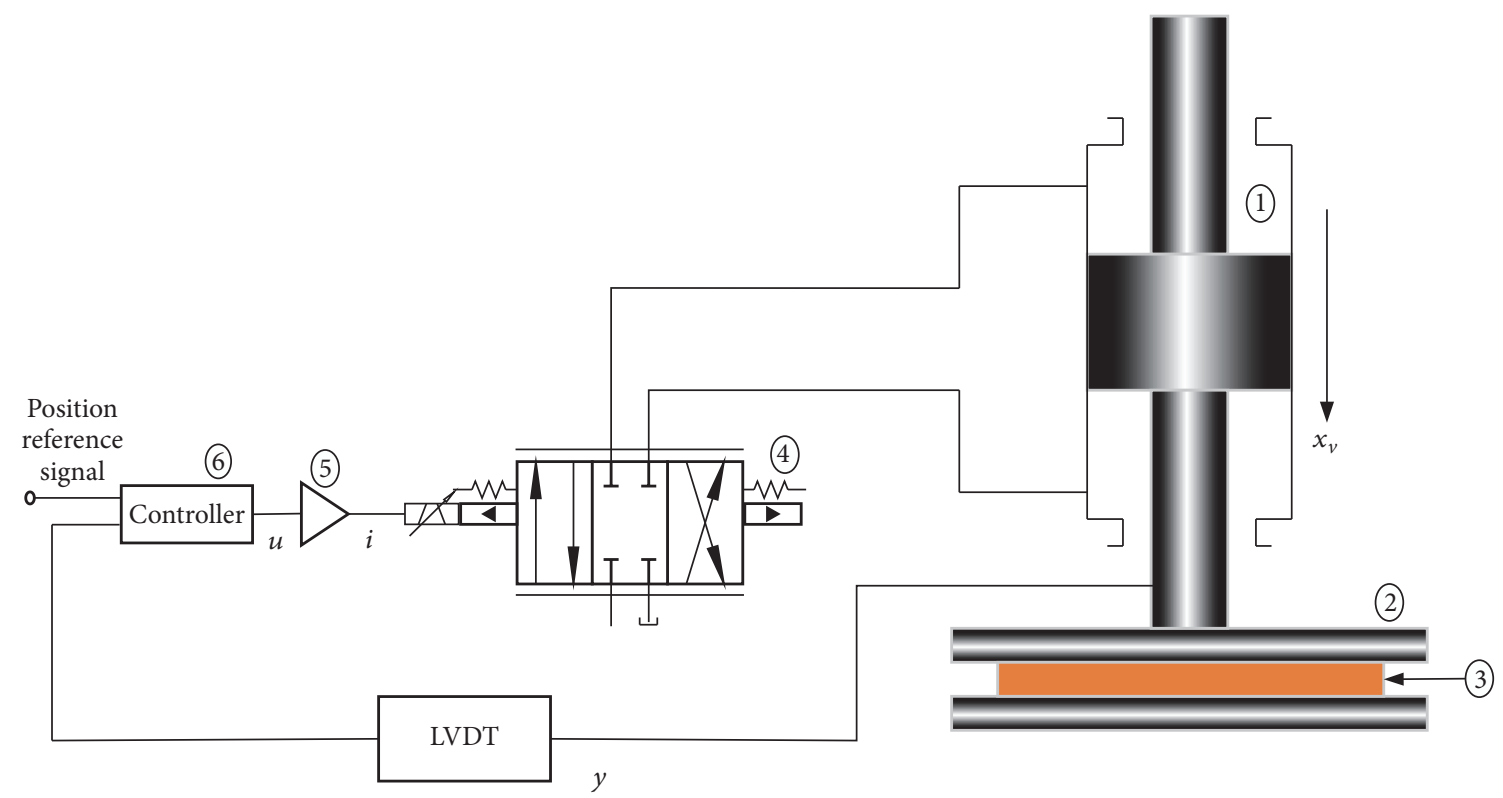

FIGURE 2: Configuration of the EHSS with a single cylinder. 1: piston of hydraulic cylinder, 2: upper platen, 3: MDF slab, 4: servo valve, 5: servo amplifier, and 6: signal comparator.

where $f(x)=a_{1} x_{1}+a_{2} x_{2}+a_{3} x_{3}, a_{1}=-4 \beta_{e} C_{\mathrm{tc}} K / m V, a_{2}=$ $-K / m-4 \beta_{e}\left(A^{2}+C_{\mathrm{tc}} B_{c}\right) / m V, a_{3}=-B_{c} / m-4 \beta_{e} C_{\mathrm{tc}} / V_{t}, a_{4}=$ $4 A \beta_{e} c_{d} \omega K_{\mathrm{sv}} K_{a} / m V_{t} \sqrt{\rho}, f\left(x_{v}\right)=\sqrt{p_{s}-p_{L} \operatorname{sgn}\left(x_{v}\right)}, g\left(x_{v}\right)=$ $a_{4} f\left(x_{v}\right)$, and $d=-\dot{F} / m-\left(4 \beta_{e} C_{\text {tc }} / m V_{t}\right) F$.

As mentioned above, the uncertainties and input saturation are considered in this paper. Due to the existence of temperature and pressure, some model parameters vary as time goes by, such as $\beta_{e}$ and $\rho$. The external load force stems from the steam pressure in the MDF slab. Therefore, parameters $a_{i}, i=1,2,3,4$ and external disturbance $d$ in (3) are uncertain. Moreover, the control input in (3) is subject to saturation nonlinearity described as follows:

$$
u(v(t))=\operatorname{sat}(v(t))= \begin{cases}\operatorname{sign}(v(t)) u_{M} & |v(t)| \geq u_{M} \\ v(t) & |v(t)| \leq u_{M},\end{cases}
$$

where $u_{M}$ is a bound of $u(t)$.

Remark 1. It is noticed that there exists a sharp corner at point $v(t)=u_{M}$, denoting nonsmoothness at this point. Hence, the DSC technique cannot be applied in theory.

Inspired by [15], using a smooth function approximates the saturation function (4) with the help of a hyperbolic tangent function. The smooth function is defined as

$$
l(v)=u_{M} \tanh \left(\frac{v}{u_{M}}\right)=u_{M} \frac{e^{v / u_{M}}-e^{-v / u_{M}}}{e^{v / u_{M}}-e^{-v / u_{M}}}
$$

For example, choose control input $v(t)=20 \sin (2 t)$ and $u_{M}=5$. Figure 3 shows approximation result of the smooth function.

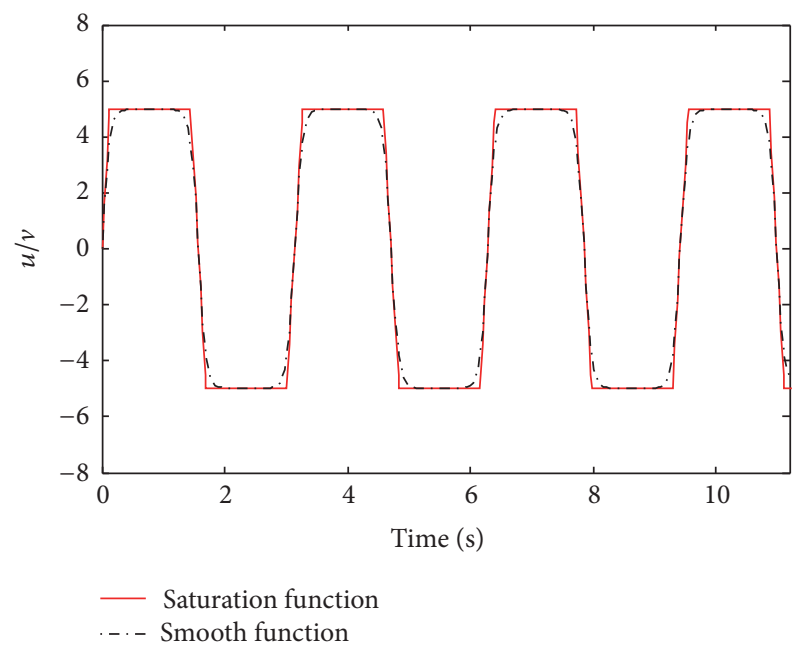

Figure 3: Approximation result of the smooth function.

Thus, the third equation of system model (3) can be rewritten as

$$
\dot{x}_{3}=f(x)+\Delta f(x)+\left[g\left(x_{v}\right)+\Delta g\left(x_{v}\right)\right] l(v)+d,
$$

where $\Delta f(x)=\Delta a_{1} x_{1}+\Delta a_{2} x_{2}+\Delta a_{3} x_{3}$ and $\Delta g\left(x_{v}\right)=$ $\Delta a_{4} f\left(x_{v}\right)$.

It is convenient for deducing the control law to define a function as follows [16]:

$$
\delta(v)=l(v)-v .
$$

Then, the smooth function can be express as

$$
l(v)=\delta(v)+v .
$$




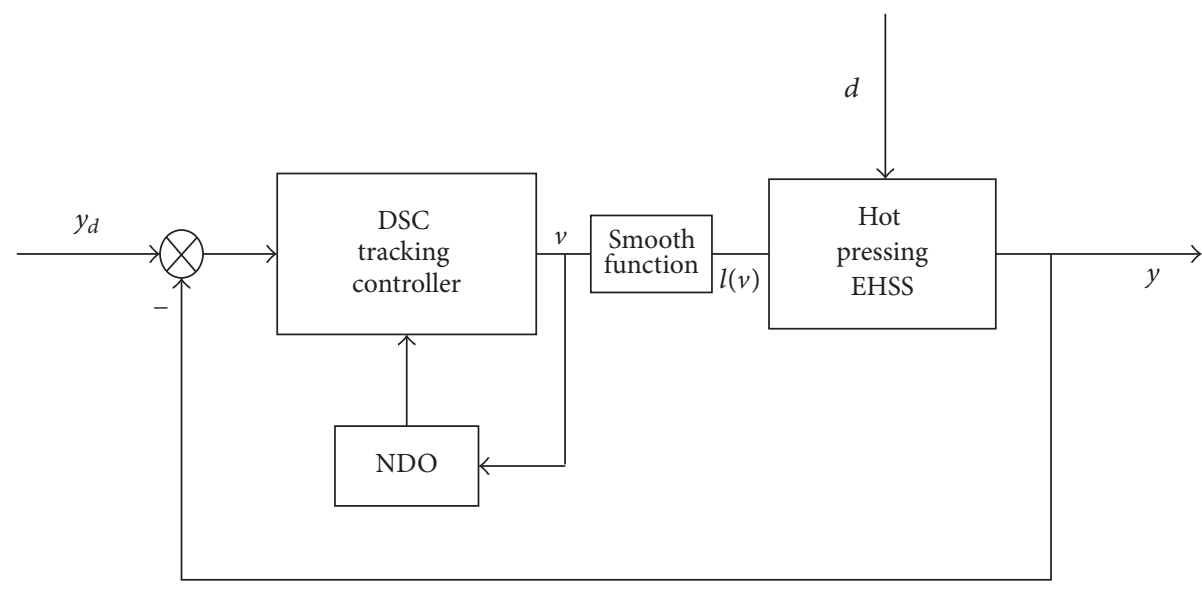

FIgure 4: Configuration of the compound controller.

Finally, the EHSS mathematic model with uncertainties and saturation is transformed into

$$
\begin{aligned}
& \dot{x}_{1}=x_{2} \\
& \dot{x}_{2}=x_{3} \\
& \dot{x}_{3}=f(x)+g\left(x_{v}\right) v+D \\
& y=x_{1},
\end{aligned}
$$

where the composite disturbance term $D=\Delta f(x)+\Delta g\left(x_{v}\right) v+$ $\left[g\left(x_{v}\right)+\Delta g\left(x_{v}\right)\right] \delta(v)+d$.

Remark 2. We may have found that the composite disturbance term is made up of parameter uncertainties, external disturbance, and saturation nonlinearities. By virtue of a NDO, the term can be estimated and fed back to the control law.

\section{A Compound Controller Design}

3.1. Overview of the Compound Controller. The compound controller is consisting of two parts, each with its own objective. The first part is to establish a tracking controller with the DSC approach. By two first-order low-pass filters, the derivatives of virtual control inputs are obtained, avoiding the "explosion of differential terms" phenomenon in the existing backstepping. The other is to suppress the influence of uncertainties and saturation in the EHSS. NDO is introduced into the control law to estimate and compensate the composite disturbance term defined above. The configuration is shown in Figure 4.

3.2. DSC Design Procedure. Similar to the backstepping technique, the design with the DSC approach is divided into three steps. In each step, a virtual input is designed for the next subsystem. However, the virtual control input is sent to first-order low-pass filters, getting a new variable and its derivative. Then, the new variable is applied to the tracking error of the next subsystem. Finally, the control law of the system is obtained in the third step. Before the procedure, tracking errors of three steps are defined as

$$
\begin{aligned}
& e_{1}=x_{1}-y_{d} \\
& e_{2}=x_{2}-x_{2 d} \\
& e_{3}=x_{3}-x_{3 d},
\end{aligned}
$$

where $x_{i}(i=1,2,3)$ is the state variable, $y_{d}$ is the desired position reference signal, and $x_{2 d}$ and $x_{3 d}$ are the outputs of the first-order low-pass filters.

Step 1. Differentiating $e_{1}$ with respect to time yields

$$
\dot{e}_{1}=\dot{x}_{1}-\dot{y}_{d}=x_{2}-\dot{y}_{d} \text {. }
$$

The virtual control input $\bar{x}_{2}$ is designed as

$$
\bar{x}_{2}=-c_{1} e_{1}+\dot{y}_{d},
$$

where $c_{1}$ is a positive design parameter.

By a first-order low-pass filter, $\bar{x}_{2}$ passes through it, obtaining a new variable $x_{2 d}$.

$$
\begin{aligned}
x_{2 d}+\tau_{2} \dot{x}_{2 d} & =\bar{x}_{2} \\
x_{2 d}(0) & =\bar{x}_{2}(0),
\end{aligned}
$$

where $\tau_{2}$ is a time constant.

Step 2. Differentiating $e_{2}$ with respect to time yields

$$
\dot{e}_{2}=\dot{x}_{2}-\dot{x}_{2 d}=x_{3}-\dot{x}_{2 d} \text {. }
$$

The virtual control input $\bar{x}_{3}$ is designed as

$$
\bar{x}_{3}=-c_{2} e_{2}+\dot{x}_{2 d},
$$

where $c_{2}$ is a positive design parameter.

Similarly, a new variable $x_{3 d}$ is obtained by a first-order low-pass filter as follows:

$$
\begin{aligned}
x_{3 d}+\tau_{3} \dot{x}_{3 d} & =\bar{x}_{3} \\
x_{3 d}(0) & =\bar{x}_{3}(0),
\end{aligned}
$$

where $\tau_{3}$ is a time constant. 
Step 3. Differentiating $e_{3}$ with respect to time yields

$$
\dot{e}_{3}=\dot{x}_{3}-\dot{x}_{3 d}=f(x)+g\left(x_{v}\right) v+D-\dot{x}_{3 d} .
$$

If $D$ is a certain term, the control law of system (9) can be deduced as

$$
v=\frac{-f(x)+\dot{x}_{3 d}-c_{3} e_{3}-D}{g\left(x_{v}\right)},
$$

where $c_{3}$ is a positive design parameter and $\dot{x}_{3 d}$ can be invoked from (16).

Remark 3. In the existing backstepping technique, the "explosion of differential term" appears in the controller design procedure owing to the calculation of the derivatives of (12) and (15). The DSC employs the first-order low-pass filters to eliminate the phenomenon, relieving the calculation burden.

Unfortunately, the assumption above is impossible because term $D$ involves the uncertainties and input saturation. Therefore, the NDO is introduced to estimate $D$. The output of the NDO is used to feed back to the control law, suppressing the influence of uncertainties and input saturation.

3.3. Nonlinear Disturbance Observer Design. The basic idea of disturbance observer based control problem can be divided into two parts. Obviously, the first part is accomplished, stabilizing the system without consideration of the unknown disturbance term $D$ and achieving tracking performance with the DSC approach. Thereafter, a NDO is constructed to the other part, estimating $D$ and feeding it back to the control law appropriately.

Assumption 4 (see [19]). The derivative of the composite disturbance term $\dot{D}$ are bounded, satisfying

$$
|\dot{D}| \leq \kappa
$$

where the upper bounds of $\eta$ and $\kappa$ are constants which is only for analytic purpose.

Then, NDO is designed as follows:

$$
\begin{aligned}
& \widehat{D}=z+P\left(x_{3}\right) \\
& \dot{z}=-N\left(x_{3}\right) z-N\left(x_{3}\right)\left[P\left(x_{3}\right)+f(x)+g\left(x_{v}\right) v\right],
\end{aligned}
$$

where $z$ is an auxiliary design state variable in the NDO, $\widehat{D}$ is the estimation of $D$, and $N\left(x_{3}\right)$ and $P\left(x_{3}\right)$ are two functions with respect to $x_{3}$, satisfying $N\left(x_{3}\right)=\partial P\left(x_{3}\right) / \partial x_{3}$.

Remark 5. The EHSS system model parameters $a_{i}(i=1,2$, $3,4)$ and the external disturbance $d$ are uncertain but their upper bound is certain where the specific magnitude is unknown in practice. Besides, the premise of considering the input saturation is that the plant must be input-to-state stable (ISS) [15]. $v$ and $\delta(v)$ are also bounded. Hence, Assumption 4 is reasonable.
Ultimately, the control law (18) is change into

$$
v=\frac{-f(x)+\dot{x}_{3 d}-c_{3} e_{3}-\widehat{D}}{g\left(x_{v}\right)} .
$$

Remark 6. From the control law (21), we see that less design parameters are introduced in contrast with the existing control law of the continuous hot pressing EHSS. Simultaneously, we need not the requirement that the bound of the disturbance term $D$ should be known.

\section{Stability Analysis}

In this section, the closed-loop system stability of the continuous hot pressing EHSS is given via Lyapunov theory. Due to the introduction of two first-order low-pass filters and NDO, a great challenge is imposed on the stability analysis.

4.1. NDO Estimation Error Analysis. Define the NDO estimation error:

$$
\widetilde{D}=D-\widehat{D}
$$

Differentiating $\widetilde{D}$ with the respect to time yields

$$
\begin{aligned}
\dot{\widetilde{D}} & =\dot{D}-\dot{\vec{D}}=\dot{D}-\left[\dot{z}+\dot{P}\left(x_{3}\right)\right]=\dot{D} \\
& -\left\{-N\left(x_{3}\right)\left[\widehat{D}-P\left(x_{3}\right)\right]\right. \\
& \left.-N\left(x_{3}\right)\left[P\left(x_{3}\right)+f(x)+g\left(x_{v}\right) v\right]+\dot{P}\left(x_{3}\right)\right\} \\
& =\dot{D}-\left[-N\left(x_{3}\right) \widehat{D}-N\left(x_{3}\right) f(x)\right. \\
& \left.-N\left(x_{3}\right) g\left(x_{v}\right) v+N\left(x_{3}\right) \dot{x}_{3}\right]=\dot{D}-\left\{-N\left(x_{3}\right) \widehat{D}\right. \\
& -N\left(x_{3}\right) f(x)-N\left(x_{3}\right) g\left(x_{v}\right) v \\
& \left.+N\left(x_{3}\right)\left[f(x)+g\left(x_{v}\right) v+D\right]\right\}=\dot{D} \\
& -\left[-N\left(x_{3}\right) \widehat{D}+N\left(x_{3}\right) D\right]=\dot{D}-N\left(x_{3}\right) \widetilde{D} .
\end{aligned}
$$

Theorem 7. Consider the continuous hot pressing EHSS model (9) with the NDO (20). The NDO estimation error $\widetilde{D}$ is bounded within a small neighborhood of origin.

Proof. Taking the integral of (23) with respect to time yields

$$
\begin{aligned}
\widetilde{D}= & {[D(0)-\widehat{D}(0)] e^{-N\left(x_{3}\right) t} } \\
& +e^{-N\left(x_{3}\right) t} \int_{0}^{t} \dot{D}(\lambda) e^{N\left(x_{3}\right) t} d \lambda .
\end{aligned}
$$

Taking (19) into account yields

$|\widetilde{D}| \leq\left[D(0)-\widehat{D}(0)-\frac{\kappa}{N\left(x_{3}\right)}\right] e^{-N\left(x_{3}\right) t}+\frac{\kappa}{N\left(x_{3}\right)}$.

Considering (25), we can get the limit value of $\widetilde{D}$ :

$$
\lim _{t \rightarrow 0}|\widetilde{D}|=\frac{\kappa}{N\left(x_{3}\right)}
$$

Therefore, Theorem 7 is proved. 
Remark 8. From (26), it is found that $\widetilde{D}$ may decrease to a small neighborhood of origin by choosing a relatively large value of $N\left(x_{3}\right)$. However, the value cannot be too large because it may lead to chattering problem in the NDO when the disturbance term $D$ increases violently. Hence, we need to select a proper function $P\left(x_{3}\right)$.

4.2. Closed-Loop System Stability Analysis. Firstly, let us do some definitions and calculations before the stability proof.

The filtering error of two first-order low-pass filters $y_{2}$ and $y_{3}$ are defined as

$$
\begin{aligned}
& y_{2}=x_{2 d}-\bar{x}_{2} \\
& y_{3}=x_{3 d}-\bar{x}_{3} .
\end{aligned}
$$

Substituting (12), (13), and (27) into (11) gets

$$
\begin{aligned}
\dot{e}_{1} & =x_{2}-\dot{y}_{d}=e_{2}+x_{2 d}-\dot{y}_{d}=e_{2}+y_{2}+\bar{x}_{2}-\dot{y}_{d} \\
& =e_{2}+y_{2}-c_{1} e_{1} .
\end{aligned}
$$

Similarly,

$$
\dot{e}_{2}=e_{3}+y_{3}-c_{2} e_{2} \text {. }
$$

Substituting (21) and (22) into (17) yields

$$
\begin{aligned}
\dot{e}_{3}= & f(x)+g\left(x_{v}\right)\left[\frac{-f(x)+\dot{x}_{3 d}-c_{3} e_{3}-\widehat{D}}{g\left(x_{v}\right)}\right]+D \\
& -\dot{x}_{3 d}=-c_{3} e_{3}+\widetilde{D} .
\end{aligned}
$$

Differentiating $y_{2}$ with the respect to time yields

$$
\begin{aligned}
\dot{y}_{2} & =\dot{x}_{2 d}-\dot{\bar{x}}_{2}=\frac{\bar{x}_{2}-x_{2 d}}{\tau_{2}}+c_{1} \dot{e}_{1}-\ddot{y}_{d} \\
& =-\frac{y_{2}}{\tau_{2}}+c_{1}\left(e_{2}+y_{2}-c_{1} e_{1}\right)-\ddot{y}_{d} \\
& =-\frac{y_{2}}{\tau_{2}}+B_{2}\left(e_{1}, e_{2}, y_{2}, \ddot{y}_{d}\right) .
\end{aligned}
$$

The derivative of $y_{3}$ can be obtained in the same way, yielding

$$
\dot{y}_{3}=-\frac{y_{3}}{\tau_{3}}+B_{3}\left(e_{2}, e_{3}, y_{3}, \ddot{x}_{2 d}\right)
$$

Define sets as follows:

$$
\begin{aligned}
& \Omega_{1}=\left\{\left(y_{d}, \dot{y}_{d}, \ddot{y}_{d}\right): y_{d}^{2}+\dot{y}_{d}^{2}+\ddot{y}_{d}^{2} \leq \vartheta\right\} \\
& \Omega_{2}=\left\{\sum_{i=1}^{3} e_{i}^{2}+\sum_{i=2}^{3} y_{i}^{2}+\widetilde{D}^{2} \leq 2 p\right\},
\end{aligned}
$$

where $\Omega_{1} \in \mathbf{R}^{3}, \Omega_{2} \in \mathbf{R}^{6}$, and $\Omega_{1} \times \Omega_{2} \in \mathbf{R}^{9}$.

Assumption 9 (see [17]). On set $\Omega_{1} \times \Omega_{2} \in \mathbf{R}^{9}$, the upper bound of $B_{i}(i=2,3)$ is defined as $M_{i}(i=2,3)$, satisfying $|B|_{i} \leq M_{i}$.
Theorem 10. In terms of the continuous hot pressing EHSS model (9) by the NDO (20) and control law (21), there exist the DSC design parameters $c_{i}(i=1,2,3)$ and the time constants of first-order low-pass filters $\tau_{i}(i=2,3)$ to guarantee that all signals of the closed-loop system are semiglobally uniformly ultimately bounded and the tracking error converges to zero asymptotically.

Proof. A set of Lyapunov candidate are chosen as

$$
\begin{aligned}
& V_{1}=\frac{1}{2} e_{1}^{2}+\frac{1}{2} e_{2}^{2}+\frac{1}{2} e_{3}^{2} \\
& V_{2}=\frac{1}{2} y_{2}^{2}+\frac{1}{2} y_{3}^{2} \\
& V_{3}=\frac{1}{2} \widetilde{D}^{2} \\
& V=V_{1}+V_{2}+V_{3} .
\end{aligned}
$$

Differentiating $V_{1}$ and invoking (28), (29), and (30) yield

$$
\begin{aligned}
\dot{V}_{1}= & e_{1} \dot{e}_{1}+e_{2} \dot{e}_{2}+e_{3} \dot{e}_{3} \\
= & e_{1}\left(e_{2}+y_{2}-c_{1} e_{1}\right)+e_{2}\left(e_{3}+y_{3}-c_{2} e_{2}\right) \\
& +e_{3}\left(-c_{3} e_{3}+\widetilde{D}\right) \\
= & -c_{1} e_{1}^{2}-c_{2} e_{2}^{2}-c_{3} e_{3}^{2}+e_{1} e_{2}+e_{2} e_{3}+e_{1} y_{2}+e_{2} y_{3} \\
& +e_{3} \widetilde{D} .
\end{aligned}
$$

Differentiating $V_{2}$ and invoking (31) and (32) yield

$$
\begin{aligned}
\dot{V}_{2} & =y_{2} \dot{y}_{2}+y_{3} \dot{y}_{3} \\
& =y_{2}\left(-\frac{y_{2}}{\tau_{2}}+B_{2}\right)+y_{3}\left(-\frac{y_{3}}{\tau_{3}}+B_{3}\right) \\
& =-\frac{y_{2}^{2}}{\tau_{2}}+B_{2} y_{2}-\frac{y_{3}^{2}}{\tau_{3}}+B_{3} y_{3} .
\end{aligned}
$$

Differentiating $V_{3}$ and invoking (23) yield

$$
\dot{V}_{3}=\widetilde{D} \dot{\bar{D}}=\widetilde{D}\left(\dot{D}-N\left(x_{3}\right) \widetilde{D}\right)=\widetilde{D} \dot{D}-N\left(x_{3}\right) \widetilde{D}^{2}
$$

Thus, from (35), (36), and (37), we can obtain

$$
\begin{aligned}
\dot{V}= & \dot{V}_{1}+\dot{V}_{2}+\dot{V}_{3} \\
= & -c_{1} e_{1}^{2}-c_{2} e_{2}^{2}-c_{3} e_{3}^{2}+e_{1} e_{2}+e_{2} e_{3}+e_{1} y_{2}+e_{2} y_{3} \\
& +e_{3} \widetilde{D}-\frac{y_{2}^{2}}{\tau_{2}}+B_{2} y_{2}-\frac{y_{3}^{2}}{\tau_{3}}+B_{3} y_{3}+\widetilde{D} \dot{D} \\
& -N\left(x_{3}\right) \widetilde{D}^{2}
\end{aligned}
$$




$$
\begin{aligned}
\leq & -c_{1} e_{1}^{2}-c_{2} e_{2}^{2}-c_{3} e_{3}^{2}-\frac{y_{2}^{2}}{\tau_{2}}-\frac{y_{3}^{2}}{\tau_{3}}+\frac{1}{2}\left(e_{1}^{2}+e_{2}^{2}\right) \\
& +\frac{1}{2}\left(e_{2}^{2}+e_{3}^{2}\right)+\frac{1}{2}\left(e_{1}^{2}+y_{2}^{2}\right)+\frac{1}{2}\left(e_{2}^{2}+y_{3}^{2}\right) \\
& +\frac{1}{2}\left(e_{3}^{2}+\widetilde{D}^{2}\right)+\frac{1}{2}\left(B_{2}^{2} y_{2}^{2}+1\right)+\frac{1}{2}\left(B_{3}^{2} y_{3}^{2}+1\right) \\
& +\frac{1}{2}\left(\widetilde{D}^{2}+\kappa^{2}\right)-N\left(x_{3}\right) \widetilde{D}^{2} \\
= & -\left(c_{1}-1\right) e_{1}^{2}-\left(c_{2}-\frac{3}{2}\right) e_{2}^{2}-\left(c_{3}-1\right) e_{3}^{2} \\
& -\left(\frac{1}{\tau_{2}}-\frac{1}{2} B_{2}^{2}\right) y_{2}^{2}-\left(\frac{1}{\tau_{3}}-\frac{1}{2} B_{3}^{2}\right) y_{3}^{2} \\
& -\left[N\left(x_{3}\right)-\frac{1}{2}\right] \widetilde{D}^{2}+\frac{1}{2} \kappa^{2}+1 .
\end{aligned}
$$

The parameters are designed as follows:

$$
\begin{aligned}
c_{1} & \geq 1+r, \\
c_{2} & \geq \frac{3}{2}+r, \\
c_{3} & \geq 1+r, \\
N\left(x_{3}\right) & \geq \frac{1}{2}+r, \\
\frac{1}{\tau_{2}} & \geq \frac{1}{2} M_{2}^{2}+\frac{1}{2}+r, \\
\frac{1}{\tau_{3}} & \geq \frac{1}{2} M_{3}^{2}+\frac{1}{2}+r,
\end{aligned}
$$

where $r$ is a positive constant.

Substituting (39) into (38) yields

$$
\begin{aligned}
\dot{V} \leq & -r e_{1}^{2}-r e_{2}^{2}-r e_{3}^{2}-r \widetilde{D}^{2}-\left(\frac{1}{2} M_{2}^{2}-\frac{1}{2} B_{2}^{2}-r\right) y_{2}^{2} \\
& -\left(\frac{1}{2} M_{3}^{2}-\frac{1}{2} B_{3}^{2}-r\right) y_{3}^{2}+\frac{1}{2} \kappa^{2}+1 \\
= & -r e_{1}^{2}-r e_{2}^{2}-r e_{3}^{2}-\left(\frac{M_{2}^{2}}{2 B_{2}^{2}} B_{2}^{2}-\frac{1}{2} B_{2}^{2}-r\right) y_{2}^{2} \\
& -\left(\frac{M_{3}^{2}}{2 B_{3}^{2}} B_{3}^{2}-\frac{1}{2} B_{3}^{2}-r\right) y_{3}^{2}+\frac{1}{2} \kappa^{2}+1 \\
= & -r e_{1}^{2}-r e_{2}^{2}-r e_{3}^{2}-r \widetilde{D}^{2}-r y_{2}^{2}-r y_{3}^{2} \\
& -\frac{1}{2} B_{2}^{2}\left(\frac{M_{2}^{2}}{B_{2}^{2}}-1\right) y_{2}^{2}-\frac{1}{2} B_{3}^{2}\left(\frac{M_{3}^{2}}{B_{3}^{2}}-1\right) y_{3}^{2} \\
& +\frac{1}{2} \kappa^{2}+1 .
\end{aligned}
$$

Considering $|B|_{i} \leq M_{i}$, we have $B_{i}^{2} / M_{i}^{2}-1 \leq 0$. Hence,

$$
\begin{aligned}
\dot{V} & \leq-r e_{1}^{2}-r e_{2}^{2}-r e_{3}^{2}-r \widetilde{D}^{2}-r y_{2}^{2}-r y_{3}^{2}+\frac{1}{2} \kappa^{2}+1 \\
& =-2 r V+\zeta
\end{aligned}
$$

where $\zeta=(1 / 2) \kappa^{2}+1$.

Satisfying $r>\zeta / 2 p$, where $p>0$ is one of the bounds of $V$, yields

$$
\dot{V} \leq-2 \cdot \frac{\zeta}{2 p} \cdot p+\zeta=0 .
$$

According to the theorem in [30], we can conclude that the closed-loop system is semiglobally stable.

Taking the integral of (41), we can get

$$
V \leq \frac{1}{2 r}+\left[V(0)-\frac{1}{2 r}\right] e^{-2 r t}
$$

Based on (43), it shows that all signals of the closed-loop system are semiglobally uniformly ultimately bounded on set $\Theta$ :

$$
\Theta=\left\{z_{1}, z_{2}, z_{3}, y_{2}, y_{3}, \widetilde{D}\right\} .
$$

By adjusting the DSC design parameters $c_{i}(i=1,2,3)$ and the time constants $\tau_{i}(i=2,3)$ of first-order low-pass filters, the set can be changed to be arbitrarily small, and the tracking error $e_{1}$ can converge to zero asymptotically finally.

\section{Simulation Results}

For the MDF continuous hot pressing EHSS, simulation study is to validate the effectiveness of proposed strategy based on Matlab2014a/Simulink. According to the practical continuous hot pressing process in the fixing-thickness phase, with temperature and pressure in a reasonable range, the nominal parameters are given as follows:

$$
\begin{aligned}
& K_{\mathrm{sv}} /\left(\mathrm{m} \cdot \mathrm{A}^{-1}\right)=0.01 \\
& K_{a} /\left(\mathrm{A} \cdot \mathrm{V}^{-1}\right)=0.0125 \\
& \omega /(\mathrm{m})=0.025 \\
& c_{d}=0.61 \\
& p_{s} /(\mathrm{MPa})=25 \\
& p_{L}=2 p_{s} / 3 \\
& \rho /\left(\mathrm{kg} \cdot \mathrm{m}^{-3}\right)=850 \\
& A /\left(\mathrm{m}^{2}\right)=0.1256 \\
& \beta_{e} /(\mathrm{MPa})=685 \\
& F /(\mathrm{N})=54780 \\
& C_{t} /\left(10^{-16} \mathrm{~m}^{5} \cdot \mathrm{N}^{-1} \cdot \mathrm{s}^{-1}\right)=5 \\
& B_{c} /\left(10^{6} \mathrm{~N} \cdot \mathrm{s} \cdot \mathrm{m}^{-1}\right)=2.25 \\
& K /\left(\mathrm{GN} \cdot \mathrm{m}^{-1}\right)=2.4 . \\
& m /(\mathrm{kg})=1 \times 10^{3} . \\
& V_{t} /\left(10^{-3} \mathrm{~m}^{3}\right)=2.356
\end{aligned}
$$


Next, the assumptions in the numerical simulation are given as follows:

(1) The control input $u(t)$ subject to function (4) where $u_{M}=8 \mathrm{~V}$ represents control input voltage of the continuous hot pressing EHSS. In the proposed strategy, a smooth function is used to approximate $u(t)$.

(2) The parameter perturbations are $\Delta a_{i}=0.1 a_{i} \sin (\pi t)$, $(i=1,2,3,4)$.

(3) The external load force is $F=54780+4000 \sin (4 \pi t)$.

(4) The initial states are $x_{1}(0)=x_{2}(0)=x_{3}(0)=0$.

The composite controller parameters are selected as follows:

(1) The DSC tracking controller parameters are chosen as $c_{1}=c_{2}=c_{3}=160$ and $\tau_{2}=\tau_{3}=0.01$.

(2) The NDO design parameter is chosen as $P\left(x_{3}\right)=$ $1000 x_{3}$ and $N\left(x_{3}\right)=1000$.

In this section, two kinds of position tracking reference signal are applied to the continuous hot pressing EHSS which are a sinusoidal signal and a constant signal. Due to the fact that the sinusoidal signal is fluctutated as time goes by, it can testify the antisaturation ability of the proposed controller effectively. On the other hand, a constant signal is used to simulate the MDF slab thickness error properly in practice.

Case 1 (tracking a sinusoidal signal). To illustrate the antisaturation ability and the robustness of the proposed method, the sinusoidal signal is set as $y_{d}=0.0005 \sin (10 \pi t)$. In this case, the initial conditions of the first-order low-pass filters are $x_{2 d}(0)=0.016$ and $x_{3 d}(0)=2.513$ by the preceding calculation. To further illustrate the superiority of the proposed method, we provided conventional sliding mode controller for the MDF continuous hot pressing EHSS. Simulation results are shown from Figures 5-8.

Clearly, the MDF continuous hot pressing EHSS with the composite controller can track the reference signal accurately. Compared with the DSC controller, the tracking error decreases into a small neighborhood of origin in less than $0.1 \mathrm{~s}$ (Figure 5).

The estimation result of the NDO is displayed in Figure 6. We can see the precise estimation of the NDO. Although the composite disturbance term $D$ varies as the time goes by rapidly, the NDO reflects the favorable estimation ability. Accordingly, the output of the NDO can be fed back to the control law, enhancing the robustness of the closed-loop system.

In Figure 7 , the control inputs are all restricted. In contrast, the control input with the method presented in this paper is continuous differential by the smooth function to handle the saturation. Moreover, the magnitude of the control input is lower than that with the traditional DSC controller. To some extent, the composite controller consumes lower power energy. Output of the first-order low-pass filters and the virtual control input curve are shown in Figure 8. We may observe the reliable capability of using the first-order
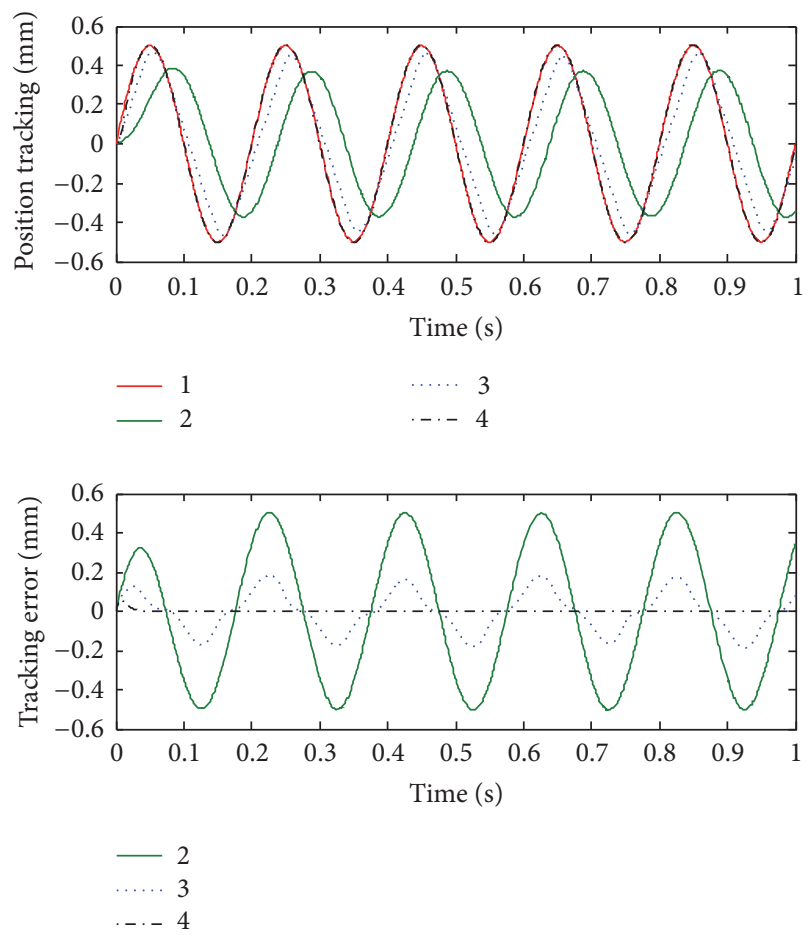

FIGURE 5: Position tracking and tracking error curve. (1 represents the desired reference signal; 2 represents sliding mode controller; 3 represents traditional DSC controller; and 4 represents the proposed strategy.)

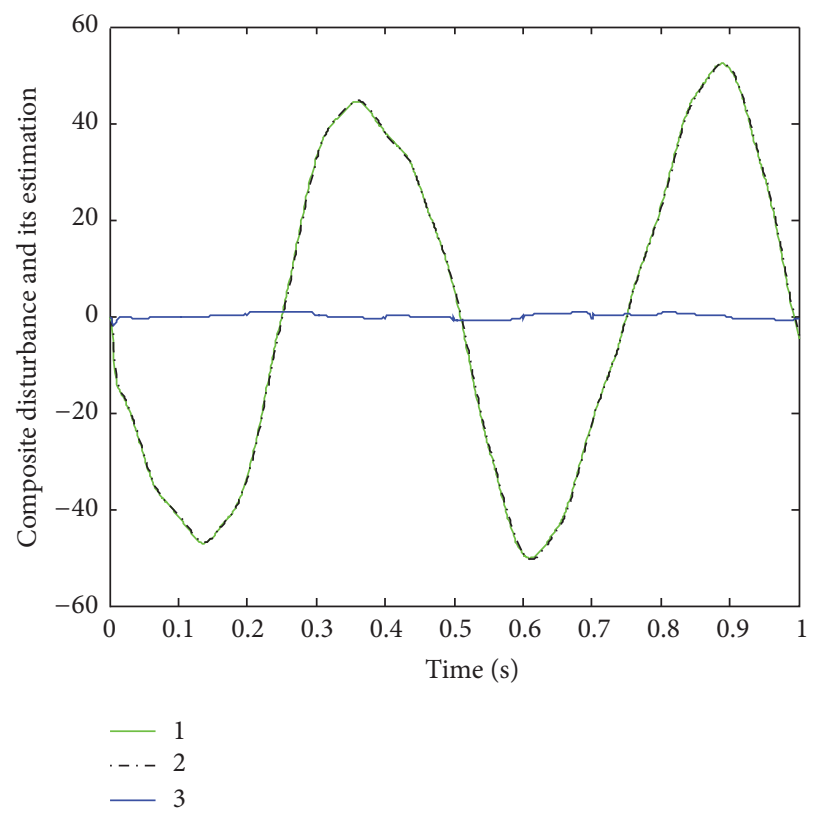

FIGURE 6: The NDO estimation curve. (1 represents the composite disturbance term; 2 represents the estimation result of the NDO; and 3 represents the estimation error.)

low-pass filter to calculate the derivative of the virtual control input.

Case 2 (tracking a constant signal). In the above case, we have demonstrated the robustness and resistance of the input saturation in the proposed method in detail. Taking the 


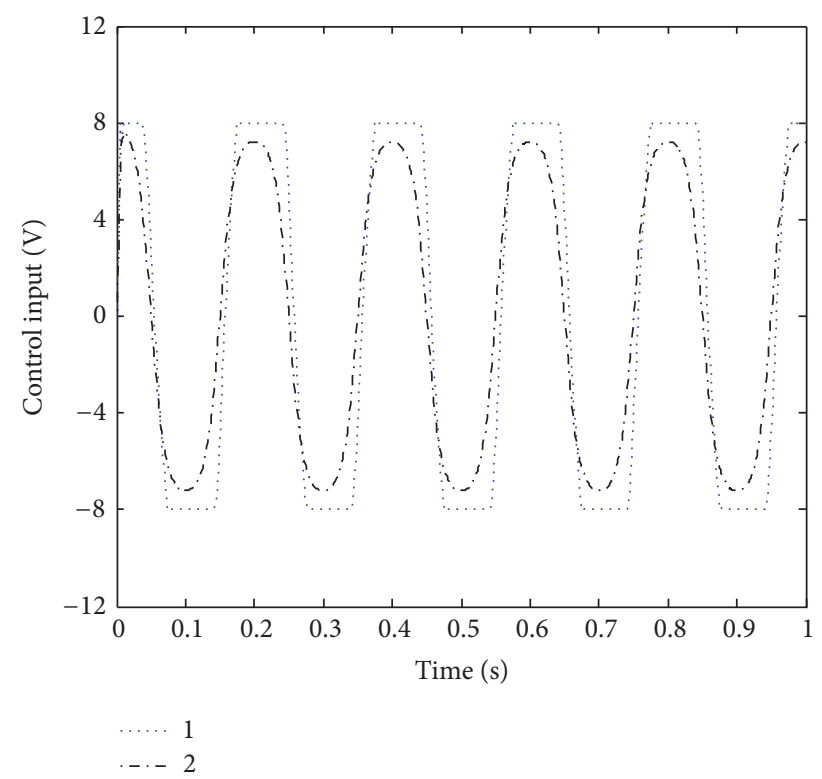

FIGURE 7: Control input curve. (1 represents the traditional DSC controller and 2 represents the proposed strategy.)
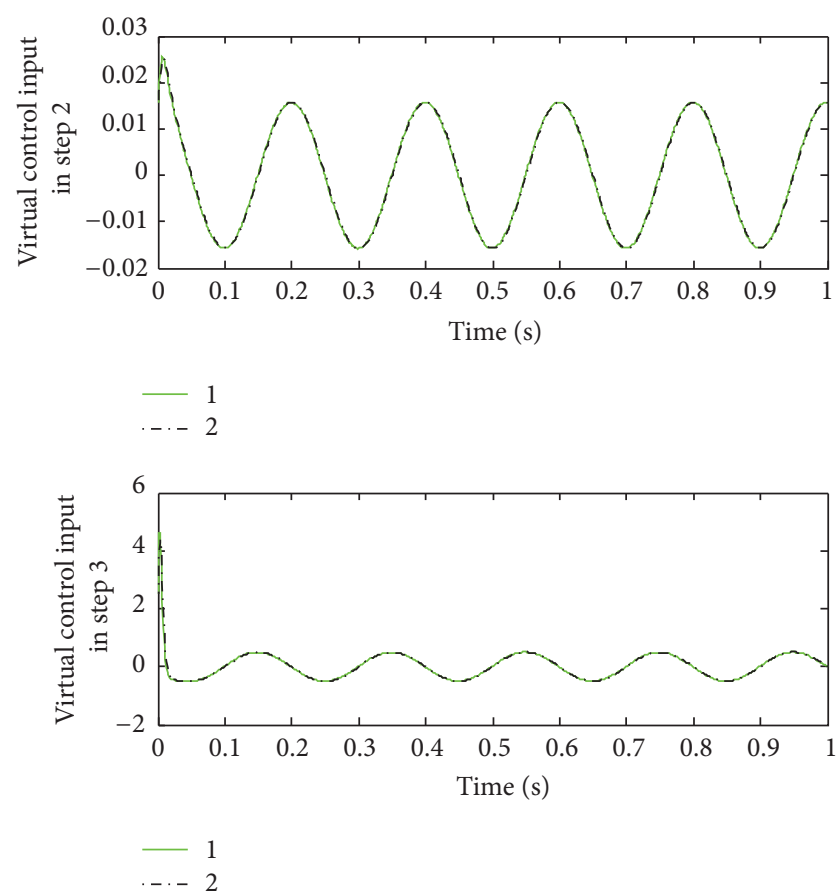

FIGURE 8: Virtual control input curve and output curve of first-order low-pass filter. (1 represents the designed virtual control input and 2 represents the output of first-order low-pass filters.)

practical continuous hot pressing process into account, the constant reference signal should be observed to simulate the thickness error.

Assuming that initial position of hydraulic cylinder is $x_{0}=0 \mathrm{~mm}$, the MDF slab thickness error is $0.5 \mathrm{~mm}$. Hence, the tracking reference signal is $y_{d}=0.5 \mathrm{~mm}$. In this case,

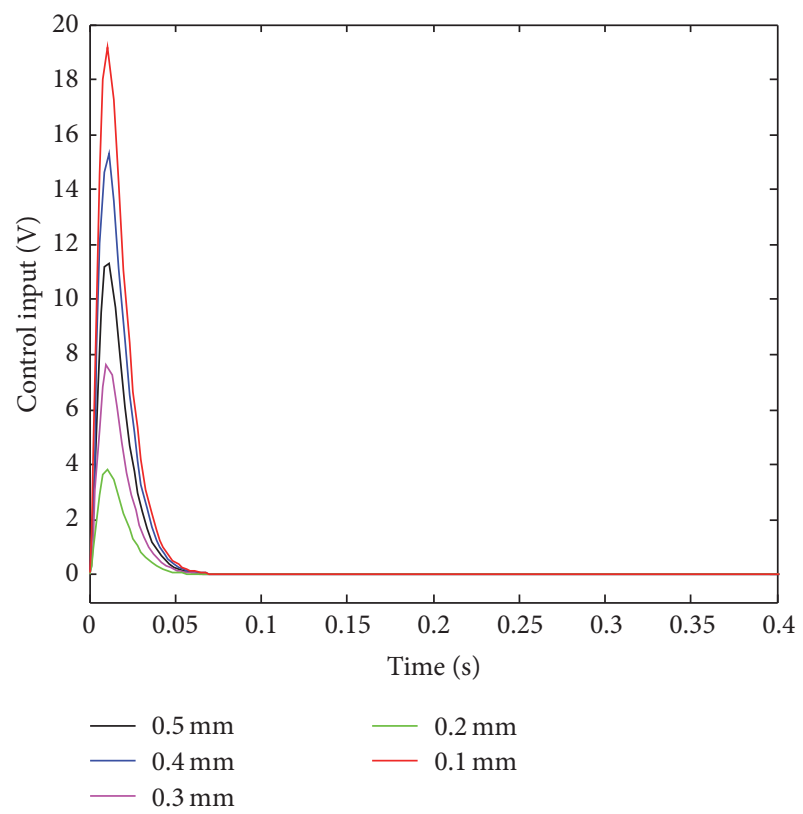

FIGURE 9: Control input curve in the condition of the different MDF slab thickness error.

the initial conditions of the first-order low-pass filters are $x_{2 d}(0)=0.08$ and $x_{3 d}(0)=12.3$, respectively. From an engineering view, Figure 9 reflects that the control input voltage rises as the MDF thickness tracking error increases. But, the actuator voltage is limited in $u_{M}=8 \mathrm{~V}$. Once the control input exceeds the upper voltage, the actuator action is invariable. Then, the MDF thickness cannot be guaranteed.

Obviously, the existence of uncertainties and input saturation in the MDF continuous hot pressing has an undesirable impact on the tracking precision and convergence time. The overshoot phenomenon emerges in the position tracking curve from Figure 10. It is notable that the overshoot should be avoided because the hot pressing in the fixing-phase is an irreversible process. Compared with the DSC controller, the proposed controller can realize the accurate tracking, achieving the objective of gauge thickness control. The tracking error is converged to a small value which can be ignored in practice.

The control input is shown in Figure 11. A relatively smooth and less saturated control input is applied for the MDF continuous hot pressing EHSS. Meanwhile, it reflected that the introduction of the NDO has an advantage in robustness.

\section{Conclusion}

In this paper, a compound control strategy is investigated for Medium Density Fiberboard (MDF) continuous hot pressing electrohydraulic servo system (EHSS) with uncertainties and input saturation. By dynamic surface control (DSC) approach, a tracking controller is constructed with less burdensome calculation burden and more flexible framework. Introducing a nonlinear disturbance observer (NDO), the control performance is improved, enhancing 

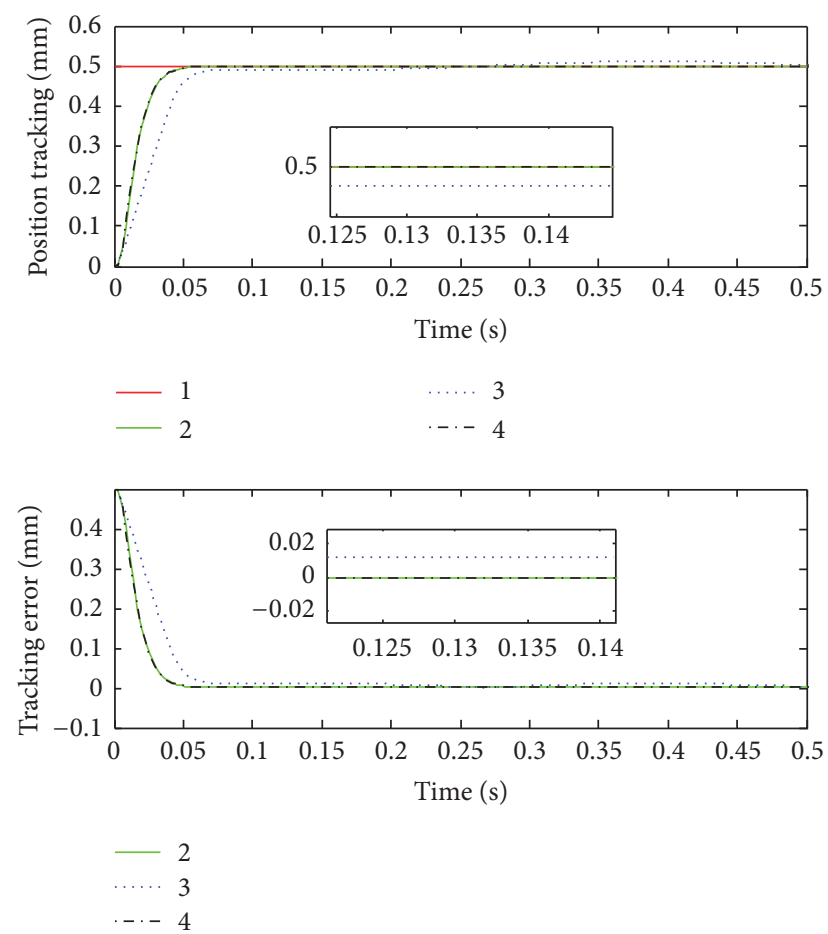

FIGURE 10: Position tracking and tracking error curve. (1 represents the desired constant signal; 2 represents the DSC controller with no input saturation and uncertainties; 3 represents the DSC in presence of uncertainties and input saturation; and 4 represents the developed compound controller.)

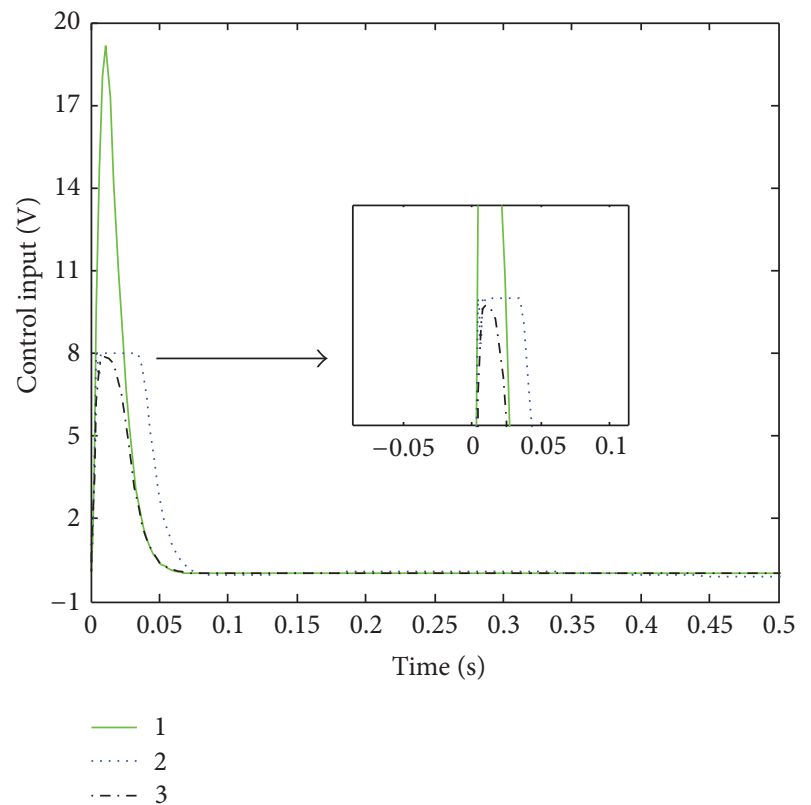

FIGURE 11: Control input curve. (1 represents control input without limitation; 2 represents DSC controller input without NDO; and 3 represents the proposed method.)

the robustness and suppressing the saturation nonlinearities. Simultaneously, fewer design parameters are required using the compound controller. Simulation results show that the proposed method may accomplish the tracking control in presence of uncertainties and input saturation successfully. The control system possesses advantages such as shorter convergence time, high control precision, and smooth control input to ensure the MDF obtained gauge thickness.

\section{Competing Interests}

The authors declare that there is no conflict of interests regarding the publication of this paper.

\section{Acknowledgments}

This work is supported by 948 Project (2014-4-46), the National Natural Science Foundation of China (31370710 and 31370565), Fundamental Research Funds for the Central Universities (DL12EB04-02), and Postdoctoral Research Fund of Heilongjiang Province (LBH-Q13007).

\section{References}

[1] S. X. Tan and D. G. Zhou, Handbook of Wood Industry, China Forestry Press Publishing, Beijing, China, 2006.

[2] K. Wang, Fiber Board Volume of Wood Industrial Utility, China Forestry Press Publishing, Beijing, China, 2002.

[3] Z. Liang-Kuan, S. Xiao-Dong, L. Ya-Qiu, and C. Jun, "Adaptive backstepping sliding mode control of MDF hot press hydraulic system based on fuzzy disturbance observer," in Proceedings of the International Conference on Mechatronics and Control (ICMC '14), pp. 1125-1130, Jinzhou, China, July 2014.

[4] K. M. Grigoriadis and V. Kapila, Actuator Saturation Control, Marcel Dekker AG, New York, NY, USA, 2002.

[5] H.-M. Chen, J.-C. Renn, and J.-P. Su, "Sliding mode control with varying boundary layers for an electro-hydraulic position servo system," International Journal of Advanced Manufacturing Technology, vol. 26, no. 1-2, pp. 117-123, 2005.

[6] C. Guan and S. Pan, "Adaptive sliding mode control of electrohydraulic system with nonlinear unknown parameters," Control Engineering Practice, vol. 16, no. 11, pp. 1275-1284, 2008.

[7] O. Cerman and P. Hušek, "Adaptive fuzzy sliding mode control for electro-hydraulic servo mechanism," Expert Systems with Applications, vol. 39, no. 11, pp. 10269-10277, 2012.

[8] Z. Wang, S. Q. Zhu, Q. C. Chen, X. Zhang, and Y. Song, "Sliding mode control of electro-hydraulic servo system for lower-limb exoskeleton based on RBF neural network," in Proceedings of the IEEE 10th Conference on Industrial Electronics and Applications (ICIEA '15), pp. 79-83, Auckland, New Zealand, June 2015.

[9] I. Ursu, F. Ursu, and F. Popescu, "Backstepping design for controlling electrohydraulic servos," Journal of the Franklin Institute, vol. 343, no. 1, pp. 94-110, 2006.

[10] C. Kaddissi, J.-P. Kenné, and M. Saad, "Identification and realtime control of an electrohydraulic servo system based on nonlinear backstepping," IEEE/ASME Transactions on Mechatronics, vol. 12, no. 1, pp. 12-22, 2007.

[11] N. M. Tri, D. N. C. Nam, H. G. Park, and K. K. Ahn, “Trajectory control of an electro hydraulic actuator using an iterative backstepping control scheme," Mechatronics, vol. 29, pp. 96-102, 2015. 
[12] N. Kapoor, A. R. Teel, and P. Daoutidis, "An anti-windup design for linear systems with input saturation," Automatica, vol. 34, no. 5, pp. 559-574, 1998.

[13] V. Adetola, D. Dehaan, and M. Guay, "Adaptive model predictive control for constrained nonlinear systems," Systems \& Control Letters, vol. 58, no. 5, pp. 320-326, 2009.

[14] L. Sonneveldt, Q. P. Chu, and J. A. Mulder, "Nonlinear flight control design using constrained adaptive backstepping," Journal of Guidance, Control, and Dynamics, vol. 30, no. 2, pp. 322336, 2007.

[15] C. Wen, J. Zhou, Z. Liu, and H. Su, "Robust adaptive control of uncertain nonlinear systems in the presence of input saturation and external disturbance," IEEE Transactions on Automatic Control, vol. 56, no. 7, pp. 1672-1678, 2011.

[16] J. Ma, S. S. Ge, Z. Zheng, and D. Hu, "Adaptive NN control of a class of nonlinear systems with asymmetric saturation actuators," IEEE Transactions on Neural Networks and Learning Systems, vol. 26, no. 7, pp. 1532-1538, 2015.

[17] D. Swaroop, J. K. Hedrick, P. P. Yip, and J. C. Gerdes, "Dynamic surface control for a class of nonlinear systems," IEEE Transactions on Automatic Control, vol. 45, no. 10, pp. 1893-1899, 2000.

[18] M. Chen, W. H. Chen, and Q. X. Wu, "Adaptive fuzzy tracking control for a class of uncertain MIMO nonlinear systems using disturbance observer," Science China Information Sciences, vol. 57, no. 1, pp. 1-13, 2014.

[19] W.-H. Chen, "Disturbance observer based control for nonlinear systems," IEEE/ASME Transactions on Mechatronics, vol. 9, no. 4, pp. 706-710, 2004.

[20] A. Mohammadi, M. Tavakoli, H. J. Marquez, and F. Hashemzadeh, "Nonlinear disturbance observer design for robotic manipulators," Control Engineering Practice, vol. 21, no. 3, pp. 253-267, 2013.

[21] J. Yang, S. Li, C. Sun, and L. Guo, "Nonlinear-disturbanceobserver-based robust flight control for airbreathing hypersonic vehicles," IEEE Transactions on Aerospace \& Electronic Systems, vol. 49, no. 2, pp. 1263-1275, 2013.

[22] J. Huang, S. Ri, L. Liu, Y. Wang, J. Kim, and G. Pak, "Nonlinear disturbance observer-based dynamic surface control of mobile wheeled inverted pendulum," IEEE Transactions on Control Systems Technology, vol. 23, no. 6, pp. 2400-2407, 2015.

[23] J. Wu, J. Huang, Y. Wang, and K. Xing, "Nonlinear disturbance observer-based dynamic surface control for trajectory tracking of pneumatic muscle system," IEEE Transactions on Control Systems Technology, vol. 22, no. 2, pp. 440-455, 2014.

[24] Y. M. T. Elobaid, J. Huang, and Y. Wang, "Nonlinear disturbance observer based robust tracking control of pneumatic muscle," Mathematical Problems in Engineering, vol. 2014, Article ID 872093, 8 pages, 2014.

[25] L. Hongyi, Z. Chen, L. Wu, and H.-K. Lam, "Event-triggered control for nonlinear systems under unreliable communication links," IEEE Transactions on Fuzzy Systems, 2016.

[26] H. Li, L. Wang, and H. Du, "Adaptive fuzzy backstepping tracking control for strict-feedback systems with input delay," IEEE Transactions on Fuzzy Systems, 2016.

[27] H. Li, Y. Gao, P. Shi, and H. K. Lam, "Observer-based fault detection for nonlinear systems with sensor fault and limited communication capacity," IEEE Transactions on Automatic Control, vol. 61, no. 9, pp. 2745-2751, 2016.

[28] Y. Zhang and L. G. Zhang, Fiber Board Manufacturing, China Forestry Press Publishing, Beijing, China, 2012.
[29] Z. S. Wu, Hydraulic Control System, Higher Education Press, Beijing, China, 2008.

[30] A. Petros and J. Sun, Robust Adaptive Control, Prentice-Hall Press, New York, NY, USA, 1996. 


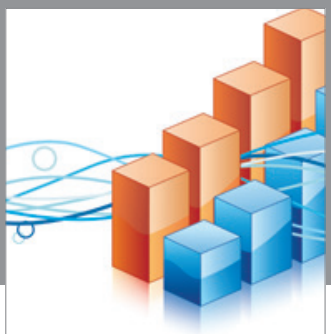

Advances in

Operations Research

vatem alat4

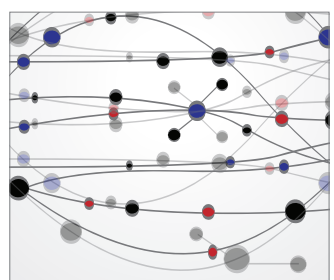

\section{The Scientific} World Journal
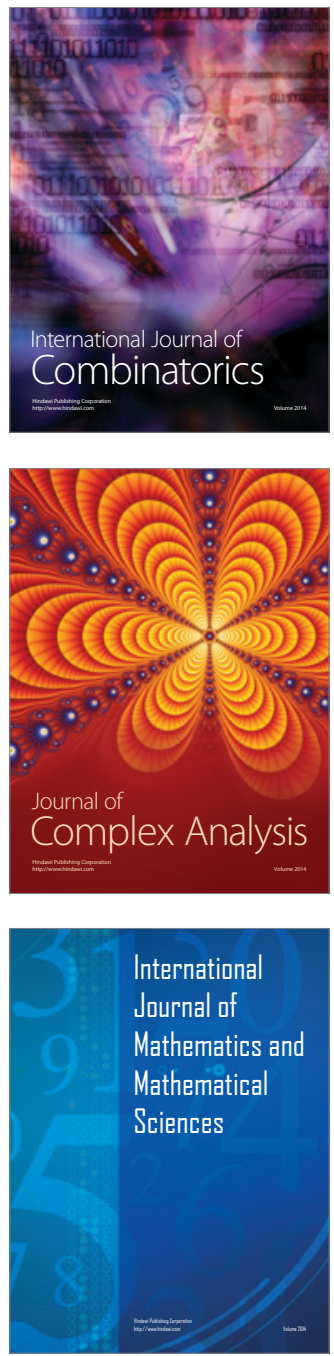
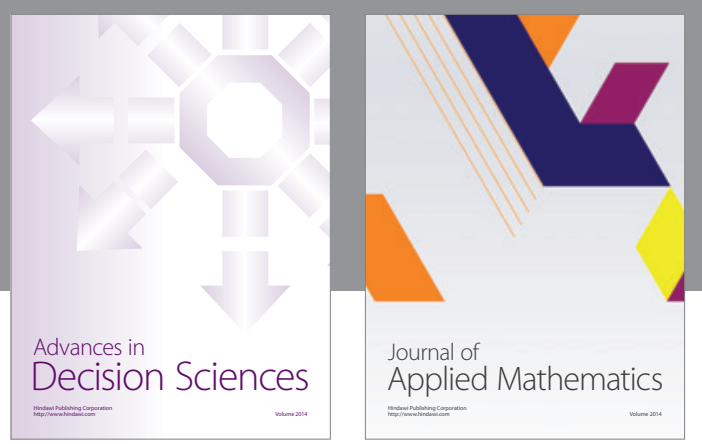

Algebra

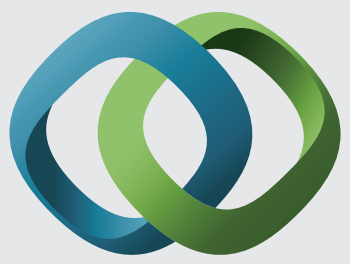

\section{Hindawi}

Submit your manuscripts at

http://www.hindawi.com
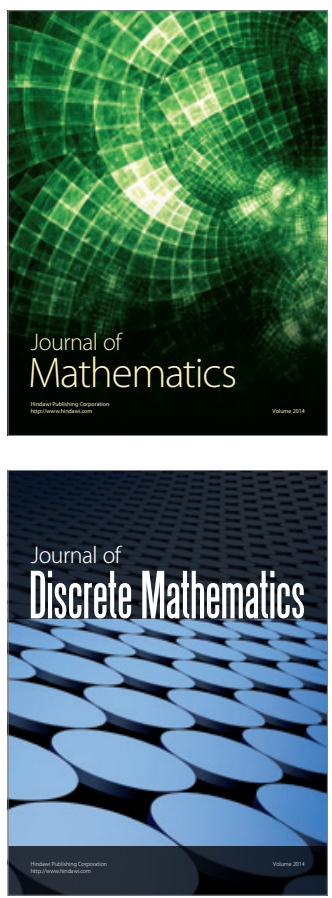

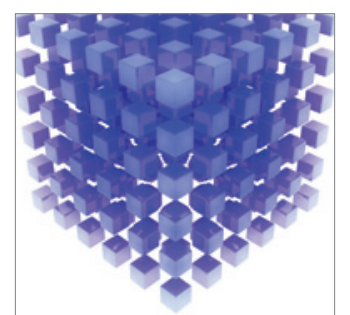

Mathematical Problems in Engineering
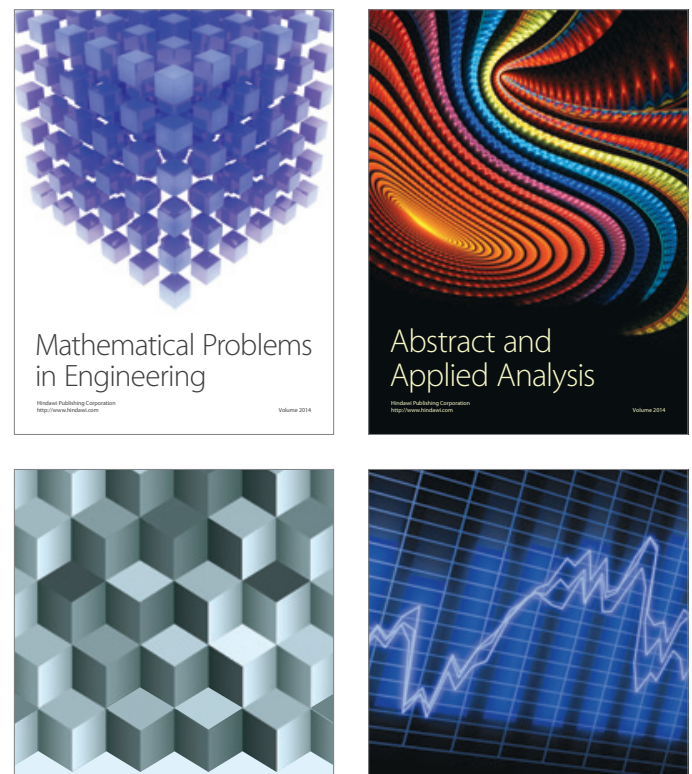

Journal of

Function Spaces

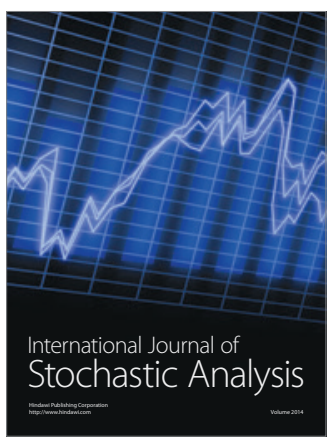

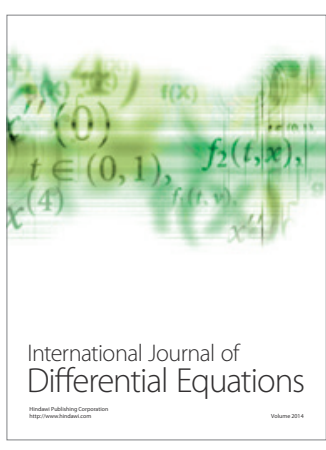
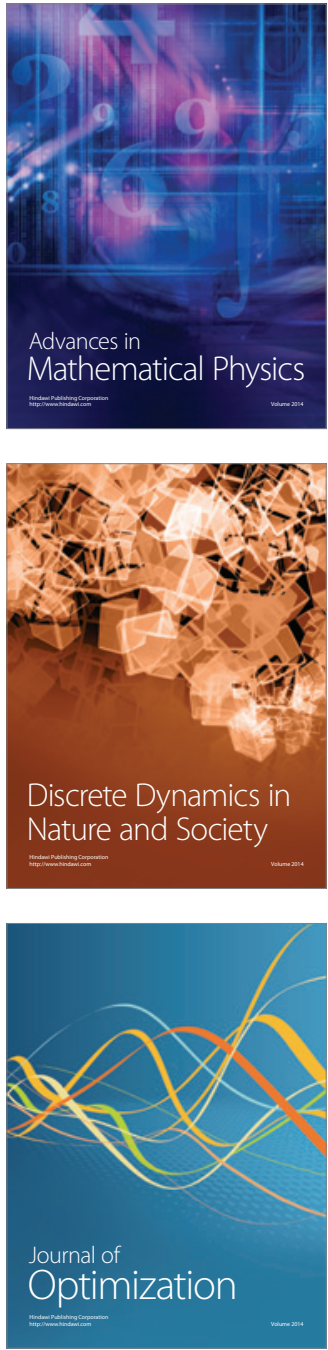